\title{
Subdiffusion with External Time Modulation
}

\author{
R. WOJNAR
}

IPPT PAN, Świętokrzyska 21, 00-049 Warszawa, Poland

To Professors Dietrich Stauffer and Marcel Ausloos on their anniversaries

A subdiffusion process, similar to a Zeldovich-Kompaneets heat conduction process, is defined by a nonlinear diffusion equation in which the diffusion coefficient takes the form $D=a(t) f^{n}$, where $a=a(t)$ is an external time modulation, $n$ is a positive constant, and $f=f(x, t)$ is a solution to the nonlinear diffusive equation. It is shown that a Zeldovich-Kompaneets solution satisfies the subdiffusion equation if $a=a(t)$ is replaced by the mean value of $a$. Also, a solution to the subdiffusion equation is constructed that may be useful in description of biological, social, and financial processes.

PACS numbers: 05.40.Jc, 05.70.Ln, 82.39.Rt, 87.18.Hf, 89.65.Gh, 89.65.Lm

\section{Introduction}

Diffusion with constant coefficient is an idealised process. A nonlinear process in which the diffusion coefficient is a power of probability density and for small values of probability the diffusion is vanishing, represents a subdiffusion.

Let $j$ denote a flux of Brownian particle diffusion. According to the Fick law $j=-D \partial f / \partial x$, where $f=f(x, t)$ is the distribution function which depends on position $x$ and time $t$. A nonlinear process in which the diffusion coefficient takes the form

$$
D=a f^{n}
$$

where $a$ and $n$ are constants, called the Zeldovich-Kompaneets subdiffusion as it is analogous to a nonlinear heat flow discussed by those authors in $1950[1,2]$. Such dependence of $D$ on $f$ leads for $n>0$ to limited diffusion without tails, that is to a subdiffusion.

In the present paper a subdiffusion process is discussed in which the coefficient $a$ in Eq.(1) is a prescribed function of time

$$
a=a(t) .
$$

Such variation of $a$ denotes modulation of diffusive process by external reasons. 
For example, it may reflect seasonal changes of moisture or nutrients in biology or societal changes following fashions and trends in the economy.

\section{Zeldovich-Kompaneets diffusion with time modulation}

Let us consider one-dimensional nonlinear diffusion

$$
\frac{\partial f}{\partial t}=\frac{\partial}{\partial x}\left(D \frac{\partial f}{\partial x}\right)
$$

where the coefficient $D$ is given by (1) and (2). Thus the equation of diffusion reads

$$
\frac{\partial f}{\partial t}=a \frac{\partial}{\partial x}\left(f^{n} \frac{\partial f}{\partial x}\right)
$$

The dimension of $f$ is $[f]=1 \mathrm{~cm}$, the dimension of $\frac{\partial}{\partial x}\left(f^{n} \frac{\partial f}{\partial x}\right)$ is $1 / \mathrm{cm}^{n+3}$. Because the dimension of the left-hand side is $(1 / \mathrm{s})(1 / \mathrm{cm})$, the dimension $[a]=\mathrm{cm}^{n+2}(1 / \mathrm{s})$. Let us introduce a non-dimensional variable

$$
\xi=\frac{x}{A^{\frac{1}{2+n}}}
$$

where

$$
A=A(t)=\int_{0}^{t} a(\tau) \mathrm{d} \tau
$$

and look for the solution in the form

$$
f=\frac{1}{A^{\frac{1}{2+n}}} \varphi(\xi) .
$$

As $\partial \xi / \partial t=-(a \xi) /[(2+n) A]$ and $\partial \xi / \partial x=1 / A^{\frac{1}{2+n}}$ we get subsequently

$$
\frac{\partial f}{\partial t}=-\frac{1}{2+n} \frac{a}{A^{\frac{1}{2+n}+1}}\left(\varphi+\xi \frac{\partial \varphi}{\partial \xi}\right), \quad \frac{\partial f}{\partial x}=\frac{1}{A^{\frac{2}{2+n}}} \frac{\partial \varphi}{\partial \xi}
$$

and

$$
\frac{\partial^{2} f}{\partial x^{2}}=\frac{1}{A^{\frac{3}{2+n}}} \frac{\partial^{2} \varphi}{\partial \xi^{2}} .
$$

Further

$$
\frac{\partial}{\partial x}\left(\varphi^{n} \frac{\partial \varphi}{\partial x}\right)=\frac{1}{A^{\frac{n+3}{2+n}}} \frac{\partial}{\partial \xi}\left(\varphi^{n} \frac{\partial \varphi}{\partial \xi}\right) .
$$

These relations permit to reduce Eq. (4) to the form

$$
\frac{\partial}{\partial \xi}\left[(2+n) \varphi^{n} \frac{\partial \varphi}{\partial \xi}+\xi \varphi\right]=0 .
$$

This ordinary differential equation has a solution

$$
\varphi=\left[\frac{n}{2(2+n)}\left(\xi_{0}^{2}-\xi^{2}\right)\right]^{1 / n},
$$

where $\xi_{0}$ is a constant of integration. For $n>0$ this formula gives the probability density distribution in the interval between the points $x= \pm x_{0}$ corrresponding at every instant to the equations $\xi= \pm \xi_{0}$ : 


$$
x_{0}=A^{\frac{1}{2+n}} \xi_{0}
$$

with $A$ given by (6). Outside the interval $f=0$. If coefficient $a$ in (1) is a constant, then $A=a t$ and the diffusion density expands with time as $x_{0} \propto t^{1 /(2+n)}$.

\section{Initial value problem}

Let at the initial instant $t=0$, the diffunding particle be at $x=0$ while $f(x, 0)=0$ elsewhere. Thus

$$
f(x, 0)=\delta(x)
$$

and at subsequent instants the density distribution spreads according to (7) and (9). The integration constant $\xi_{0}$ in (9) is given by normalization

$$
\int_{-x_{0}}^{x_{0}} f \mathrm{~d} x=\frac{1}{A^{\frac{1}{2+n}}}\left[\frac{n}{2(2+n)}\right]^{1 / n} \int_{-x_{0}}^{x_{0}}\left(\xi_{0}^{2}-\xi^{2}\right)^{1 / n} \mathrm{~d} x=1,
$$

which leads to appearing the Euler beta function $B(x, y)$. We get

$$
\int_{-x_{0}}^{x_{0}}\left(\xi_{0}^{2}-\xi^{2}\right)^{1 / n} \mathrm{~d} x=\xi_{0}^{\frac{2}{n}} x_{0} \int_{0}^{1} t^{-\frac{1}{2}}(1-t)^{\frac{1}{n}} \mathrm{~d} t=\xi_{0}^{\frac{2}{n}} x_{0} B\left(\frac{1}{2}, 1+\frac{1}{n}\right),
$$

which inserted into (12) gives

$$
\xi_{0}^{2+n}=2\left(\frac{2}{n}+1\right) B^{-n}\left(\frac{1}{2}, 1+\frac{1}{n}\right) .
$$

Hence expression (7) takes the form

$$
f=\frac{1}{x_{0}} \frac{1}{B\left(\frac{1}{2}, 1+\frac{1}{n}\right)}\left(1-\frac{x^{2}}{x_{0}^{2}}\right)^{1 / n} .
$$

Function $B$ can be written in terms of the Euler $\Gamma$ function

$$
B\left(\frac{1}{2}, 1+\frac{1}{n}\right)=\frac{\Gamma\left(\frac{1}{2}\right) \Gamma\left(1+\frac{1}{n}\right)}{\Gamma\left(\frac{1}{2}+1+\frac{1}{n}\right)} .
$$

From (13) we obtain

$$
\xi_{0}^{2+n}=2^{1-n}(2+n)^{1+n} \frac{\Gamma^{n}\left(\frac{1}{2}+\frac{1}{n}\right)}{n \pi^{n / 2} \Gamma^{n}\left(\frac{1}{n}\right)},
$$

where relations $\Gamma\left(\frac{1}{2}\right)=\sqrt{\pi}$ and $\Gamma(x+1)=x \Gamma(x)$ were used.

\section{Moments of the distribution}

The second moment or the variance is given by

$$
\sigma^{2}=\int_{-x_{0}}^{x_{0}} x^{2} f \mathrm{~d} x
$$

where $f=f(x, t)$ is given by (14). We get

$$
\sigma^{2}=\frac{n}{2+3 n} x_{0}^{2}=\frac{n}{2+3 n} A^{\frac{2}{2+n}} \xi_{0}^{2}
$$

where Eq. (10) was used. 
The fourth reduced moment or the kurtosis

$$
\kappa=\frac{1}{\sigma^{4}} \int_{-x_{0}}^{x_{0}} x^{4} f \mathrm{~d} x=3 \frac{2+3 n}{2+5 n}
$$

is always for $n>0$ less than 3 , the value for the case of linear diffusion, $n=0$.

\section{Linear diffusion}

For $n \rightarrow 0$ we have $\xi_{0} \rightarrow 2 / \sqrt{n}$. Moreover, in this limit $B\left(\frac{1}{2}, 1+\frac{1}{n}\right) \rightarrow \sqrt{\pi n}$ because $\lim _{n \rightarrow 0} \Gamma\left(\frac{1}{n}\right) / \Gamma\left(\frac{1}{n}+\frac{1}{2}\right)=\sqrt{n}$, cf. [3], and the solution given by formula (9) is

$$
f=\frac{1}{2} \frac{1}{\sqrt{\pi A}} \lim _{n \rightarrow 0}\left(1-\frac{x^{2}}{4 A} n\right)^{1 / n}=\frac{1}{2} \frac{1}{\sqrt{\pi A}} \exp \left(-\frac{x^{2}}{4 A}\right) .
$$

For $n \rightarrow 0$ we have $a \rightarrow D$ and $A \rightarrow D t$. Therefore we regain

$$
f=\frac{1}{2} \frac{1}{\sqrt{\pi D t}} \mathrm{e}^{-\frac{x^{2}}{4 D t}},
$$

which is the solution of diffusion equation for a linear case.

The variance for this case is given by (17) with $n \rightarrow 0$ and $\xi_{0} \rightarrow 2 / \sqrt{n}$. Moreover, because $a=D$ for $n=0$

$$
\sigma=\int_{-x_{0}}^{x_{0}} x^{2} f \mathrm{~d} x=2 D t
$$

It is the Einstein result for the mean square displacement in linear Brownian diffusion.

\section{Wigner semicircle distribution}

This distribution arises as the limiting distribution of eigenvalues of random symmetric matrices as the size of the matrix goes to infinity, cf. [4], and in our discussion is obtained for $n=2$. The solution (7) takes the form

$$
f=\frac{1}{2 A^{1 / 2}} \sqrt{x_{0}^{2}-x^{2}}
$$

with $x_{0}^{2}=\frac{4}{\pi} A^{1 / 2}$. For $a=$ const we have $A=D t$ and

$$
f=\frac{1}{2 \sqrt{D t}} \sqrt{\frac{4}{\pi} \sqrt{D t}-x^{2}}
$$

The variance of this function is $\sigma=x_{0}^{2} / 4=\frac{1}{\pi} A^{1 / 2}$ and the kurtosis $\kappa=2$.

\section{Discussion}

According to Eq. (17)

$$
\sigma^{2} \propto A^{\frac{2}{2+n}} .
$$

If $a=$ const, $A=(a t)^{\frac{2}{2+n}}$ and the power is always less than 1 for $n>0$, which means that we really deal with subdiffusion.

Such behaviour of limited diffusion is observed in porous media, it reveals in limited growth of colony of bacteria or clump of grass, and in another collective 
behaviours [5-7]. Diffusional mobility in the cytoplasm of living cells strongly decreases with an increasing radius of the tracked particle, and leads to cellular phenomenon known as molecular crowding [8]. In economics, investors with small capital cannot practically get in the market. Market saturation in which a product cannot be further distributed (diffused) is also known. Well determined boundaries of towns and villages in their historical development suit this model, cf. also [9].

\section{Acknowledgments}

This work was supported by the KBN grant No. 4 T07A 00327.

\section{References}

[1] Y.B. Zeldovich, A.S. Kompaneets, Collection of papers celebrating the seventieth birthday of Academician A.F. Ioffe, Ed. P.I. Lukirskii, Moskva 1950, p. 61 (in Russian).

[2] L.D. Landau, E.M. Lifshitz, Fluid Mechanics, Pergamon, Oxford 1959.

[3] I.M. Ryżyk, I.S. Gradsztejn, Table of integrals, series and products, PWN, Warszawa 1964 (in Polish).

[4] E.P. Wigner, Ann. Math. 67, 325 (1958).

[5] J.A. Shapiro, Annu. Rev. Microbiol. 52, 81 (1998).

[6] E. Ben-Jacob, I. Cohen, I. Golding, D.L. Gutnick, M. Tcherpakov, D. Helbing, I.G. Ron, Physica A 282, 247 (2000).

[7] H. Bloom, Who's smarter: chimps, baboons or bacteria? The power of group IQ, Submitted by Howard Bloom on 7 Nov. 2007 — www.scientificblogging.com.

[8] M. Weiss, M. Elsner, F. Kartberg, T. Nilssony, Biophys. J. 87, 3518 (2004).

[9] F. Michael, M.D. Johnson, Physica A 320, 525 (2003). 